Original Article

\title{
Revision of the Predictive Method Improves Precision in the Prediction of Stroke Outcomes for Patients Admitted to Rehabilitation Hospitals
}

\author{
Akiyoshi Matsugi, PhD, $\mathrm{PT}^{1)^{*}}$, Keisuke Tani, $\mathrm{PT}^{2)}$, Yasuhiro Mitani, PhD, $\mathrm{PT}^{3)}$, \\ Kosuke Oku, MS, $\mathrm{PT}^{4}$, Yoshiki Tamaru, MS, $\mathrm{OT}^{1)}$, Kiyoshi Nagano, PhD, $\mathrm{PT}^{1}$ ) \\ 1) Faculty of Rehabilitation, Shijonawate Gakuen University: 5-11-10 Hojo, Daitou City, Osaka \\ 574-0011, Japan \\ 2) Department of Rehabilitation, Baba Memorial Hospital, Japan \\ 3) Faculty of Allied Health Sciences, Kansai University of Welfare Sciences, Japan \\ 4) Department of Rehabilitation, Hanna Chuo Hospital, Japan
}

\begin{abstract}
Purpose] The purpose of this study was to confirm the accuracy of a revised method for predicting the Functional Independence Measure (FIM) at discharge when stroke patients are first admitted to a rehabilitation hospital. [Subjects and Methods] The predictive equation with logarithmic trend line was calculated based on the total score of the FIM at admission and discharge in 93 patients with cerebral infarction (CI) and 60 patients with intracerebral hemorrhage (ICH). In other patients with $\mathrm{CI}$ or ICH (validation group), the differences between the actual FIM and the predicted FIM at discharge calculated by the $\mathrm{CI}$ or ICH equation and the combined $(\mathrm{CI}+\mathrm{ICH})$ equation, as well as by the CI or ICH equation and combined equation used in a previous study, were calculated. [Results] The multiple correlation coefficients of the CI equation, ICH equation, and combined equation were 0.87 , 0.71 , and 0.8 . The residual of the actual FIM and predicted FIM at discharge calculated by the CI equation was the smallest in the CI validation group. In the ICH validation group, the residual calculated for ICH patients alone was smaller than that calculated by the previous ICH equation. [Conclusion] This easy-to-use method using a new equation for prediction was more precise than the previous equation. Therefore, we should revise the equation for predicting stroke patient outcome strata according to data from within the governing medical administration system. Key words: Prediction, Functional Independence Measure, Stroke
\end{abstract}

(This article was submitted Jan. 29, 2014, and was accepted Mar. 15, 2014)

\section{INTRODUCTION}

Predicting the outcome for the level of activities of daily living (ADL) is important in the rehabilitation of stroke patients, and various methods are used. One easy-to-use method of prediction is calculation of the initial ADL level and final ADL level ${ }^{1,2)}$. The Functional Independence Measure (FIM), which indicates the level of ADL, at discharge from the rehabilitation hospital could be predicted using the total score of the FIM at admission to a rehabilitation hospital $^{3)}$. The precision of the prediction is higher using the reciprocal of the FIM score, and the correlation coefficient of the predicted FIM and actual FIM was 0.93 in patients with cerebral infarction $(\mathrm{CI})$ or intracerebral hemorrhage $(\mathrm{ICH})^{4}$. Furthermore, the discharge FIM score can be predicted using a logarithm of the FIM scores from two initial time points after admission, and the coefficient of determi-

*Corresponding author. Akiyoshi Matsugi (E-mail: aki.pt0422@gmail.com)

(C2014 The Society of Physical Therapy Science. Published by IPEC Inc. This is an open-access article distributed under the terms of the Creative Commons Attribution Non-Commercial No Derivatives (by-ncnd) License $<$ http://creativecommons.org/licenses/by-nc-nd/3.0/> . nation was found to be $0.945^{5}$. However, when predicting it just after admission, we cannot use this method because it needs two scores for the FIM and the interval between these measurements must be more than two weeks. Thus, in this study, we confirmed whether a prediction method with a single logarithmic FIM score at admission to a rehabilitation hospital is sufficiently precise.

The predictive equation for a disease must be useful. However, a previous study revealed that the correlation coefficient of a predictive equation encompassing several diseases was lower than that of one specific to one disease ${ }^{6)}$. This result indicates that the more the variety of diseases is decreased, the greater the precision of the predictive equation. Therefore, we investigated whether the precision can be maintained by confining the equation to a representative disease, such as $\mathrm{CI}$ or ICH.

There is a very useful equation for prediction that uses a single measure, the FIM score at admission to a rehabilitation hospital. However, the periods from onset to admission to a rehabilitation hospital and the length of hospitalization are shorter than previously in Japan. This may introduce difficulties in using the same equation to predict stroke outcome because the patient strata are different. Therefore, the equation for prediction must be modified for patients with 
Table 1. Characteristics of patients with $\mathrm{CI}$ or ICH

\begin{tabular}{lcccc}
\hline & \multicolumn{3}{c}{ CI } & \multicolumn{2}{c}{ ICH } \\
\cline { 2 - 5 } & $\begin{array}{c}\text { Calculation } \\
\text { group }\end{array}$ & $\begin{array}{c}\text { Validation } \\
\text { group }\end{array}$ & $\begin{array}{c}\text { Calculation } \\
\text { group }\end{array}$ & $\begin{array}{c}\text { Validation } \\
\text { group }\end{array}$ \\
\hline Number of patients & & & & \\
$\quad$ Male & 53 & 23 & 36 & 25 \\
Female & 40 & 23 & 24 & 19 \\
Days & & & & \\
Between onset and admission & 26.2 & 25.2 & 26.5 & 29.0 \\
Length of stay & 64.6 & 62.5 & 69.8 & 71.6 \\
Total FIM score & & & & \\
At admission & 72.0 & 65.5 & 43.5 & 50.0 \\
At discharge & 87.0 & 93.0 & 77.5 & 86.0 \\
\hline
\end{tabular}

shorter periods from onset to admission to a rehabilitation hospital.

In this study, we divided each group of stroke patients into two groups, one for calculating the equation (calculation group) and the other for validating the calculated equation (validation group). We attempted to confirm whether the precision of the prediction improved when the predictive equation was calculated using only people suffering from the same disease, comparing the residuals between predictive and actual FIM scores for CI patients or ICH patients in the validation groups with those for the $\mathrm{CI}$ and ICH validation patients combined. A similar approach was taken in the previous study on $\mathrm{CI}, \mathrm{ICH}$ and $\mathrm{SAH}$ patients undertaken in Japan ${ }^{6}$, which found that equations calculated for a specific disease were more precise than those for an aggregate or "combined" group.

\section{SUBJECTS AND METHODS}

The study subjects included 243 patients who had experienced their first stroke, other than a cerebellar tentorium, and were admitted to a rehabilitation hospital during the period April 2007 to February 2010. Patients included in the study had a diagnosis of CI or ICH but had no past history of hemiplegia and were independent in daily living before admission. The patients were admitted to the hospital within 15-60 days after their stroke, and they all lived to be discharged. Patients at the rehabilitation hospital underwent physical therapy, occupational therapy, and speech therapy for one hour a day or more and more than five days a week. The ethics committee of Shijonawate Gakuen University approved all study protocols that we used the entirely coded information of patients.

The 139 patients with CI were retrospectively divided into two groups, one group for calculating the equation to predict functional outcome at discharge (calculation group) and one group to validate the equation (validation group). Two-thirds of the patients, who were randomly arranged, were assigned to the calculation group, and the remaining 46 patients were assigned to the validation group. Likewise, the 104 patients with ICH were randomly divided into a calculation group and validation group. Forty-four patients were assigned to the validation group to match the number of patients in the validation group for CI. Table 1 shows the data for these patients.

We calculated the equations of the logarithm curve for the FIM at admission and FIM at discharge, and the coefficient of determination in the $\mathrm{CI}$ and $\mathrm{ICH}$ calculation groups. These equations were defined as the $\mathrm{CI}$ equation and $\mathrm{ICH}$ equation. Furthermore, we calculated another equation and coefficient of determination for a group comprising both calculation groups combined, and the equation was defined as the combined equation.

The predictive score for the FIM at discharge of patients with CI was calculated with two equations calculated in this study and two previous equations, which were [discharge $\mathrm{FIM}=106.88 \ln ($ admission FIM $)-95.35]^{6)}$ for patients with CI, ICH, or SAH and [discharge FIM = $104.05 \ln (\mathrm{ad}-$ mission FIM) -84.03$]^{6)}$ for only patients with CI. These were defined as the previous CI equation and previous combined equations. Similarly, in ICH patients, the predictive score for the FIM at discharge of patients with ICH was calculated with two equations, which were the ICH equation and combined equation, calculated in this study and two previous equations, which were [discharge FIM $=106.88$ $\left.\ln (\text { admission FIM) }-95.35]^{6}\right)$ for patients with CI, ICH, or SAH and [discharge FIM $=102.78 \ln$ (admission FIM) $61.54]^{6)}$ for only patients with ICH. These equations were defined as the previous ICH equation and previous combined equation. The absolute value of the residuals between the predictive value and actual value were calculated, and the values were compared among the 4 equations. The Wilcoxon signed-rank sum test was conducted to test the differences in medians among the 4 equations. The alpha level was 0.05 for the statistical analysis, but Bonferroni correction was applied in multiple comparisons.

\section{RESULTS}

The calculated equations with regression and multiple correlation coefficients were discharge FIM $=50.58 \times 1 \mathrm{n}$ [admission FIM] -123.28 and 0.87 in patients with CI (CI equation), discharge FIM $=49.36 \times \ln$ [admission FIM] 111.29 and 0.71 in patients with ICH (ICH equation), and discharge FIM $=48.44 \times \ln$ [admission FIM] -111.45 and 0.8 in patients with $\mathrm{CI}$ and $\mathrm{ICH}$ (combined equation). 
Table 2. Residuals of actual FIM and predictive FIM

\begin{tabular}{lrr}
\hline & Median & $\begin{array}{c}\text { Quartile } \\
\text { deviation }\end{array}$ \\
\hline CI patients & 7.2 & 3.4 \\
CI equation & 19.8 & 11.4 \\
Previous CI equation & 10.2 & 3.9 \\
Combined equation & 14.1 & 6.0 \\
Previous combined equation & & \\
ICH patients & 10.3 & 5.7 \\
ICH equation & 39.6 & 28.9 \\
Previous ICH equation & 9.8 & 5.6 \\
Combined equation & 15.3 & 6.9 \\
Previous combined equation & & \\
\hline
\end{tabular}

The absolute values of the residuals between the predictive value and actual value in CI patients calculated by the previous CI equation, previous combined equation, $\mathrm{CI}$ equation, and combined equation were $19.8 \pm 11$ (median \pm quartile deviation), $14.1 \pm 6,7.2 \pm 3$, and $10.2 \pm 4$, respectively. There were significant differences among all equations in predicting the discharge FIM of a patient with CI $(\mathrm{p}<0.0083)$ (Table 2).

The absolute values of the residuals between the predictive value and actual value in ICH patients calculated by previous ICH equation, previous combined equation, ICH equation and combined equation were $39.6 \pm 29,15.3 \pm 7$, $10.3 \pm 6$, and $9.8 \pm 6$, respectively. The value calculated by the previous ICH equation was significantly higher than those calculated by the previous combined equation, ICH equation, and combined equation $(\mathrm{p}<0.0083)$ (Table 2$)$.

\section{DISCUSSION}

Predicting the outcome of stroke is important in rehabilitation, and various methods are used ${ }^{7-10)}$. The period from onset to admission to a rehabilitation hospital and the length of hospitalization are generally shorter in Japan. This fact makes it difficult to use an equation that has been used to predict stroke outcome elsewhere, because the patient strata differ according to medical policies. Thus, the equation for prediction in Japan needs to be modified for patients with a shortened period from onset to admission to a rehabilitation hospital.

In this study, the correlation coefficients of the predicted FIM and actual FIM in CI patients, ICH patients, and CI$\mathrm{ICH}$ patients were $0.87,0.71$, and 0.8 , and these values were higher than those of the equations calculated in a previous study. Furthermore, the residual of the actual FIM and predicted FIM calculated by our equation for CI patients was significantly smaller than that of the previous equation for CI patients. These findings indicate that the precision of prediction of the outcome for CI patients admitted to a rehabilitation hospital within approximately one month after onset becomes high using our equation. In addition, the residual of actual FIM and predicted FIM calculated by the equation for CI patients was significantly smaller than that calculated by the combined equation for $\mathrm{CI}$ and $\mathrm{ICH}$ patients, indicating that prediction becomes more precise using the equation calculated only from CI patients. The previous combined equation, which used the initial total FIM scores at admission to a rehabilitation hospital, was useful for prediction of the ADL at discharge. However, these findings suggest that we must revise the equation in accordance with the typical alterations of the patients' strata depending on the medical administration practices for their states.

On the other hand, the residual of the actual FIM and predicted FIM calculated by our equation for ICH patients was significantly smaller than that of the previous ICH equation. This finding indicates that the precision of prediction of outcome for ICH patients who are admitted to a rehabilitation hospital within approximately one month after onset becomes high using our equation. On the other hand, there was no significant difference between the residuals calculated by ICH equation and combined equation. This finding indicates that the predictive precision for patients with ICH does not increase even if the equation exclusive to $\mathrm{ICH}$ is used.

In conclusion, the precision of prediction of stroke outcome can be improved using an equation exclusive to patients with a particular disease, such as $\mathrm{CI}$ or ICH. The results of this study suggest that this easy-to-use method with a new equation for prediction is more precise than the previous equation. Therefore, physicians should revise the equation for prediction of stroke patient strata according to the governing medical administration system.

\section{REFERENCES}

1) Gladman JR, Harwood DM, Barer DH: Predicting the outcome of acute stroke: prospective evaluation of five multivariate models and comparison with simple methods. J Neurol Neurosurg Psychiatry, 1992, 55: 347-351. [Medline] [CrossRef]

2) Heinemann AW, Linacre JM, Wright BD, et al.: Prediction of rehabilitation outcomes with disability measures. Arch Phys Med Rehabil, 1994, 75: 133-143. [Medline]

3) Inouye M, Kishi K, Ikeda $Y$, et al.: Prediction of functional outcome after stroke rehabilitation. Am J Phys Med Rehabil, 2000, 79: 513-518. [Medline] [CrossRef]

4) Sonoda S, Saitoh E, Nagai S, et al.: Stroke outcome prediction using reciprocal number of initial activities of daily living status. J Stroke Cerebrovasc Dis, 2005, 14: 8-11. [Medline] [CrossRef]

5) Koyama T, Matsumoto K, Okuno T, et al.: A new method for predicting functional recovery of stroke patients with hemiplegia: logarithmic modelling. Clin Rehabil, 2005, 19: 779-789. [Medline] [CrossRef]

6) Inouye M: Predicting outcomes of patients in Japan after first acute stroke using a simple model. Am J Phys Med Rehabil, 2001, 80: 645-649. [Medline] [CrossRef]

7) Koyama T, Matsumoto K, Okuno T, et al.: Relationships between independence level of single motor-FIM items and FIM-motor scores in patients with hemiplegia after stroke: an ordinal logistic modelling study. J Rehabil Med, 2006, 38: 280-286. [Medline] [CrossRef]

8) Frank B, Schlote A, Hasenbein U, et al.: Prognosis and prognostic factors in ADL-dependent stroke patients during their first in-patient rehabilitation-a prospective multicentre study. Disabil Rehabil, 2006, 28: 13111318. [Medline] [CrossRef]

9) Frank M, Conzelmann M, Engelter S: Prediction of discharge destination after neurological rehabilitation in stroke patients. Eur Neurol, 2010, 63: 227-233. [Medline] [CrossRef]

10) Veerbeek JM, Kwakkel G, van Wegen EE, et al.: Early prediction of outcome of activities of daily living after stroke: a systematic review. Stroke, 2011, 42: 1482-1488. [Medline] [CrossRef] 